\title{
Convergence Research in the Age of Big Data: Team Science, Institutional Strategies, and Beyond
}

\author{
Daniel Sui, Vice Chancellor for Research and Innovation \\ Jim Coleman, Provost and Executive Vice Chancellor for Academic Affairs \\ University of Arkansas
}

$\mathrm{W}$ hile there have been distinct subjects for human intellectual inquires for thousands of years, discipline-based and interdisciplinary research have been with us in the academy for only the last two centuries (Fredeman et al., 2010). Disciplines covering specifically defined subject matters emerged as universities expanded in size since the Industrial Revolution and especially as universities evolved increasingly to stress research alongside teaching (Klein, 1990). Disciplines that could hire their own faculty, design their own curriculum, grant their own Ph.D.s, publish specialized journals, and hold their own annual meetings have been the driving force for the spectacular growth of both the educational and research enterprise of higher education throughout the world, especially among American Universities (Jacobs, 2013; Woeler and Millar, 2013). In the natural sciences and engineering, the hardening of disciplines was aided by industrial demand for specialized researchers. Before that time, scholars were expected to be generalists.

Ever since the emergence and growth of strong disciplines, there have been calls for interdisciplinary collaboration in the academy for both education and research (Graff, 2015). Calls in the early twentieth century for interdisciplinarity often focused on teaching. This was a reaction to the creation of the disciplinary major. The general education (GenEd) movement in the United States and elsewhere also aimed to make university education more relevant to the needs of modern citizenship. In the 1960s again, interdisciplinarity was often advocated as a means to make university education more relevant. It was widely felt that disciplines were ill-equipped to prepare students to address pressing social problems (Abbott, 2001). By the start of the twenty first century, this interest in interdisciplinary education had been matched by a growing interest in interdisciplinary research as well (NAS, 2005a; Atkinson and Crowe, 2006; Szostak, 2013).
With the explosion of Big Data during the past ten years, discussions on interdisciplinary research has entered a new phase, with a strong emphasis on convergence research through a team science approach. The goal of this paper is to present a synoptic overview on how we may facilitate convergence research in the age of big data. The rest of this paper is organized as the following. After a brief introduction, there is an overview on the general concept of convergence research and how it is different from traditional multi-, inter-, and trans-disciplinary work. The following section outlines key elements of a team science approach for conducting data-driven convergence research following the emerging fourth paradigm. The next session discusses institutional strategies, opportunities, and challenges to promote convergence research, followed by a summary and conclusion in the last section. 
Convergence Research: An Overview

Convergence is the new buzz word these days in science, business, public policy and beyond, as evidenced by over 5,000 books published recently with "convergence" as part of the book title according to Amazon.com. Inevitably, convergence means different things to different audiences despite Watson (2016) mounting convincing evidence that convergence is really at the heart of scientific progress throughout history. In the context of research, we draw primarily on the NSF's definition (https://www. integrating knowledge, methods, and expertise from different disciplines and forming novel frameworks to catalyze scientific discovery and innovation. Convergence research is closely related to other forms of research that span across different disciplines - multi-, inter-, and trans-disciplinarity (Figure 1), but also has its distinctive meaning. It is the closest to trans-disciplinary research which was historically viewed as the pinnacle of evolutionary integration across disciplines (Bergmann et al., 2012).

\section{Multi- $\rightarrow$ Inter- $\rightarrow$ Transdisciplinary}
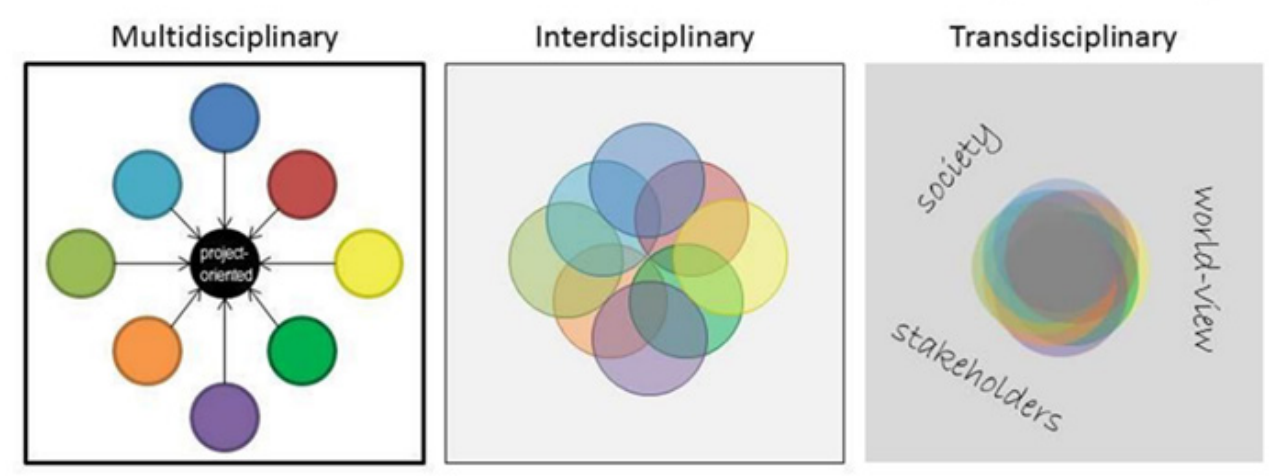

\section{- Integration: Separated $\rightarrow$ Integrated $\rightarrow$ "Become One"}

\section{Figure 1. Multi-, Inter-, and Trans-disciplinarity \\ [Source: https://nanohub.org/groups/howpeoplelearnnano/crossdisciplinary nature of_nanotechnology, fair non-commercial use via Creative Commons agreement]}

nsf.gov/od/oia/convergence/index.jsp) and the National Academies' Report on Convergence (NAS, 2014).

Growing convergence research at the U.S. National Science Foundation (NSF) was identified in 2016 as one of 10 Big Ideas for Future NSF Investments. Convergence research is a means of solving vexing research problems - in particular, complex problems focusing on societal needs (Bainbridge and Roco, 2016). Convergence research typically entails
According to NSF and NAS, convergence research must have two primary characteristics (Figure 2):

- Transdisciplinarity (Figure 1) - Deep integration across disciplines. As experts from different disciplines pursue common research challenges, their knowledge, theories, methods, data, research communities and languages become increasingly intermingled or integrated. New 
frameworks, paradigms or even disciplines can form sustained interactions across multiple communities.

- Stakeholder synergy: Research driven by a specific and compelling problem that draws together academic researchers, policy makers and industry partners. Convergence research is generally inspired by the need to address a specific challenge or opportunity, whether it arises from deep scientific questions or pressing societal needs. op holistic and robust theoretical frameworks, problem-solving strategies, and innovative ways of collaboration in new exciting areas of research.

The continuing explosion of big data (both quantitative and qualitative) during the past ten years is transforming how we can conduct research in multiple fields. We strongly believe that data-driven approach will serve as a catalyst to stimulate future convergence research and the emerging team science will play increasingly important roles in convergence research due to its mandates on transdisciplinarity and stakeholder synergy.

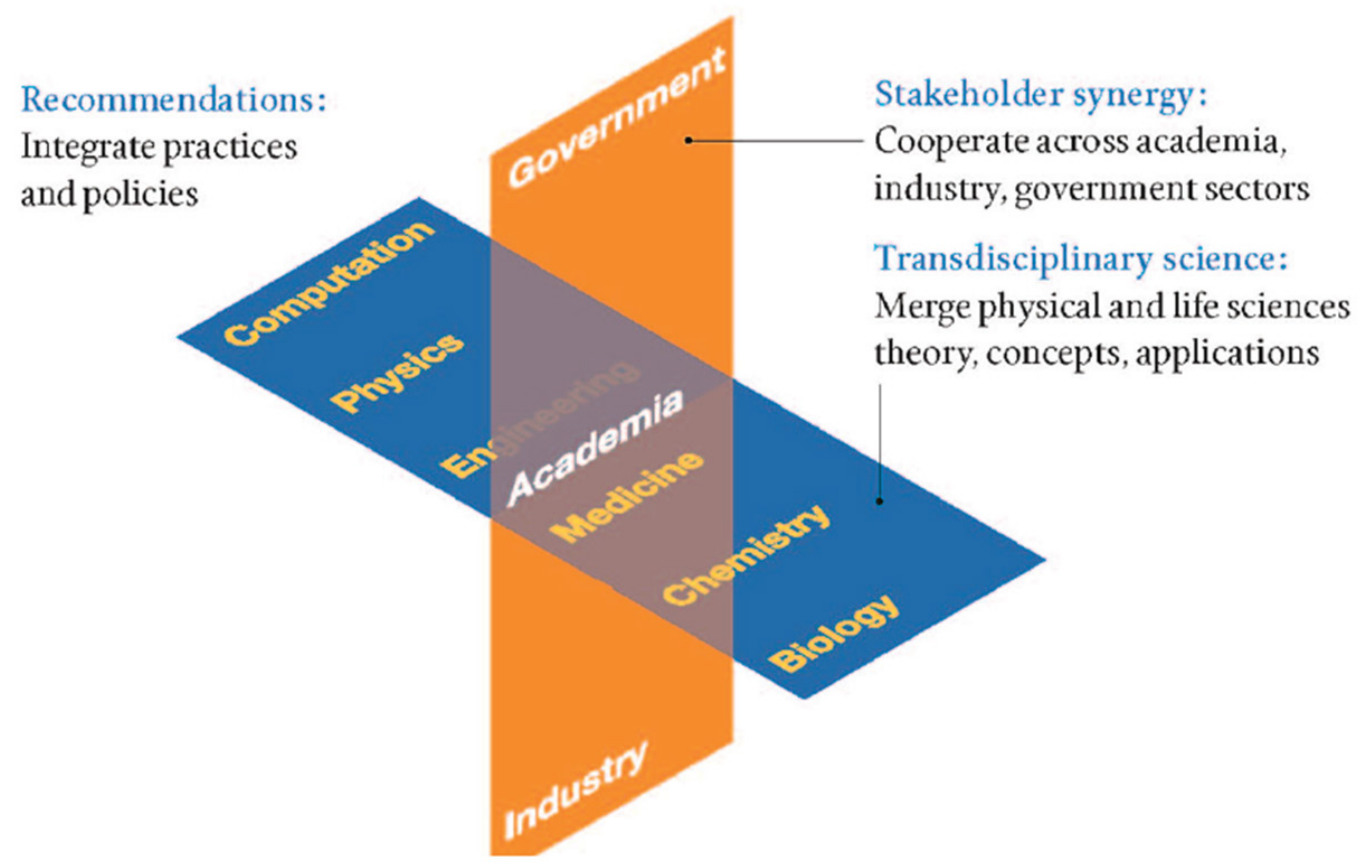

Figure 2. Two-dimensions of Convergence Research [Source: NAS, 2014]

Since its inception, the convergence paradigm intentionally brings together intellectually diverse researchers to develop effective ways of communicating and synergizing across disciplines by adopting common frameworks and a new scientific language, which may, in turn, empower researchers to devel-
Convergence Research and Big Data: A Team Science Approach

As demonstrated by the convergence research projects recently funded by NSF (https://www.nsf.gov/od/oia/ convergence/index.jsp), avariety ofdiverse approaches have been proposed and used to conduct convergence research, but two 
approaches featured prominently in convergence research - data science and team science approaches.

Transdisciplinary research by the $4^{\text {th }}$ paradigm.

According to Jim Gray at IBM (https://jimgray.azurewebsites.net), for over two thousand years, science has been conducted according to three paradigms - empirical science, theoretical science and computational science until big data exploded onto the scene. The availability of big data has transformed multiple fields, including physical sciences, medical/health sciences, engineering, social sciences, and even humanities. The emerging data-driven fourth paradigm to conduct basic research provides new opportunities to grow convergence research to a new level (Hey et al., 2010). Although data science needs new infrastructure, theoretical framework, and domain specific techniques, it is integral part and parcel of the fourth industrial revolution.

Through convergence research, the rapidly emerging field of data-intensive science (aka eScience) will continue to transform the world's scientific and computing research communities and inspire the next generation of scientists. Increasingly, scientific breakthroughs will be powered by advanced computing capabilities that help researchers manipulate and explore massive datasets. The speed at which any given scientific discipline advances will depend on how well its researchers collaborate with one another, and with technologists, in areas of eScience such as databases, workflow management, visualization, and cloud-computing technologies. The fourth paradigm of discovery based on data-intensive science offers insights into how the potential of convergence research can be fully realized.
Stakeholder synergy by team science. Stakeholder synergy - the integration of academia, industry, and government is the second defining characteristics for convergence research. To achieve stakeholder synergy, a team science approach is needed. In general, team science is a collaborative effort to address a scientific challenge that leverages the strengths and expertise of professionals trained in different fields (NAS, 2005b; Wuchty, 2007). Although traditional single investigator driven approaches are ideal for many scientific endeavors, coordinated teams of investigators with diverse skills and knowledge may be especially helpful for studies of complex social problems with multiple causes.

Over the past two decades, there has been an emerging emphasis on scientifically addressing multi-factorial problems, such as climate change, the rise of chronic disease, and the health impacts of social stratification. This has contributed to a surge of interest and investment in team science. Increasingly, scientists across many disciplines and settings are engaging in team-based research initiatives. These include small and large teams, uni- and multi-disciplinary groups, and efforts that engage multiple stakeholders such as scientists, community members, and policy makers (Fiore, 2008; Disis, 2010). Academic institutions, industry, national governments, and other funders are also investing in team science initiatives.

A growing trend within team science is cross-disciplinary science in which team members with training and expertise in different fields work together to combine or integrate their perspectives in a single research endeavor. Cross-disciplinary team science has been identified as a means to engage in expansive studies that address a broad array of complex 
and interacting variables. It is seen as a promising approach to accelerate scientific innovation and the translation of scientific findings into effective policies and practices.

In addition, the science of team science (SciTS) is a rapidly emerging field focused on understanding and enhancing the processes and outcomes of team science (Stokols et al., 2008). A key goal of SciTS is to learn more about factors that maximize the efficiency, productivity, and effectiveness of team science initiatives. A diverse group of scholars contributes to SciTS (Falk-Krzesinski et al., in press). They bring conceptual, historical, and methodological approaches from a wide variety of disciplines and fields, including public health, management, communications, and psychology. They have created measures to assess team science processes and outcomes, and to influence contextual and environmental conditions (Table 1). Applying these measures can help researchers evaluate team

\section{Table 1. Major areas of inquiry in SciTS} [Source: Stokols, 2008]

- Methods and models for the study of team science

- The structure and organization of team science, particularly the collaborative processes moderated by a variety of contextual environmental factors

- Team characteristics and dynamics, such as the elements of effective leadership, ideal team composition, and communication styles

- Design and outcomes of training programs to support team science

- Translation of team science findings to practice and policy

- Scientific and societal outcomes of team science such as scientific discoveries and innovations, knowledge dissemination, and long-term public health impacts science, improve the quality of ongoing initiatives, and develop best practices.

Among the multiple insights gained from the research in SciTS, we now know that interpersonal dynamics among team members are the key for the success of a team science project. Team members' collaborative skills and experiences can be very useful to guide our future efforts of data science-driven team science convergence research. In addition, the success of team science is influenced by a variety of contextual environmental influences (Börner et al., 2010). These factors influence each stage of a scientific initiative, with implications for efficiency, productivity, and overall effectiveness. For example, funding trends from government, industry, and private foundations can exert a huge influence on how research is being conducted. The recent emphasis by both public and private funding agencies on convergence and interdisciplinary collaborative projects addressing society's grand challenges will surely further stimulate and promote team science approaches in science (NAS, 2018). Institutional infrastructure and resources for communication and data sharing are also very important. Team processes, including the existence of agreements related to proprietary rights to data and discovery (King and Persily, 2019), as well as mechanisms for feedback and reflection, can also shape the outcome of team efforts. Last but not least, organizational policies, such as those relating to promotion and tenure, can also significantly incentivize or discourage team-based endeavors.

Institutional Strategies for Promoting Convergence Research: Opportunities and Challenges

We have seen many strategies put in place with the purpose of facilitating and stimulating convergence research and 
team science in our previous and current roles at several institutions. This has provided us with some empirical evidence of how various strategies have fared. But empirical evidence has been difficult to interpret for several reasons. Sometimes it is just simply unclear whether there have been positive results. At other times, increases in team-based research and convergence research have improved, but there is no control group that would enable one to determine whether the strategy that was implemented actually caused the improvement. For example, we have been at institutions that have created research space designed to encourage team-based, interdisciplinary, and/ or convergence research. At those institutions, the facilities were populated with some of the institution's most productive and collaborative scientists. The research programs in those facilities showed great success in productivity and collaborative research. Yet, it is impossible to answer the question whether those highly productive scientists became more collaborative, or were more productive, than they would have been had they stayed in their original facilities.

Nonetheless, we believe that there are multiple golden opportunities to conduct team-based convergence research using big data right now, but there exist certain challenges and barriers we need to overcome. We'd like to share some of those challenges, our experience with a few things that we have tried, and the University of Arkansas's institutional strategies to promote convergence research via a team-based data science approach.

\section{Opportunities.}

The new digital economy and new business models.

The fourth Industrial Revolution (Schwab, 2015) is unfolding rapidly in front of us, driven by new innovations and advances in AI, block chain, cloud computing, and data analytics. The economy will grow increasingly digital and be built upon digital platforms. Convergence research will be needed to address the pressing issues of this economy head-on. At the University of Arkansas, we have been implementing several efforts to marshal our resources in this direction including the creation of a center of excellence in block chain research, a cross-campus data science degree, and identifying convergence research in data science as an institutional investment.

New funding opportunities from government, industry, and private foundations.

The new digital economy has created new demands for data-driven convergence research. The U.S. federal agencies have all developed a new data strategy (https://strategy.data.gov). NSF has been leading the funding opportunities through its Harnessing the Data Revolution initiative (https://www.nsf.gov/cise/ harnessingdata). NIH has developed a similar data science strategic plan (https:// datascience.nih.gov/sites/default/files/ NIH_Strategic_Plan_for_Data_Science_ Final_508.pdf) with focus on infrastructure, analytic tools, data ecosystem, stewardship, and workforce development.

With the growth of new data-driven businesses and the use of data to enhance the traditional industry/business, every company/business is in the process of developing a new data strategy (https://www. forbes.com/sites/bernardmarr/2019/03/13/ why-every-company-needs-a-data-strategy-for-2019/\#3ff319e64cbb). Once again, these create new opportunities for da-ta-driven convergence research. Private foundations and non-profit organizations have also increased their investments in research related to data science and data analytics (https:// www.rockefellerfoun- 
dation.org/blog/introducing-data-science-social-impact).

Potentially major scientific breakthroughs that won't be accomplished otherwise.

We can learn a lot from the natural world, or at least find strong metaphors in the processes of organismal evolution. Ecological systems are often characterized by having edge effects. Edge effects are changes in population or community structures that occur at the boundary of two or more habitats. ${ }^{[1]}$ There is often a relative explosion of biodiversity that occurs in these areas where two or more separate eco-systems overlap. Many major advances in science occur in the area where disciplines overlap. For example, these "edge effects" in science have led to new fields such as molecular biology and biomedical engineering. We believe that major advances in the integration of big data into convergence research will also occur on the "edges" where disciplines, approaches, vocabulary, and more overlap. By facilitating interactions at the edges, institutions should be able to also facilitate advances in convergence research.

New opportunities to integrate arts, humanities, and social sciences with STEM fields.

The promotion of convergence research has further raised broader awareness that all human knowledge are branches of the same tree (NAS, 2018). We strongly believe the on-going trend towards convergence research also provides a golden opportunity to further integrate the arts, humanities, and social sciences with the traditional STEM fields, especially for those scholars practicing in the emerging digital humanities. Data-driven team science approach could potentially be added to humanity scholars' methodological repertoire (Dobson, 2019). We are fully aware of the on-going debates about the future of digital humanities (Gold and Klein, 2019) and a major cultural change needed for the practice of humanity scholarship. One thing we are absolutely convinced is that with the increasing automation and adoption of robotics in everything we do, we need to find creative ways (more than ever) to integrate the arts, humanities, and social science with STEM fields (Levit, 2018). To us, this integration will represent convergence research at its highest/deepest levels.

\section{Challenges.}

There are, of course, many challenges inherent in our institutions to fully engaging in convergence research. We list some of those below.

\section{Academic culture.}

Graduate students are generally trained to have a primary allegiance to a discipline, which carries through to the faculty years. The importance of belonging to a discipline is enforced or promulgated in several ways in universities. For example, academic departments are generally built around disciplines, partly because they may need to be for curricular reasons. Departments are not just an administrative unit, though, but become like a family unit, where members of the department work to garner resources for their unit and to foster the success of the department and hence the discipline. Furthermore, faculty often receive their most important rewards from their discipline (e.g., awards from professional societies, grants from discipline specific panels, and respect that comes from being recognized in a discipline). Also, in general, the academic culture has focused recognition of research on individuals generally for their independent research contributions (e.g., membership in the National Academies, fellows of major societies, and research recognition awards on university campuses), not to teams. 
Thus, the incentives driving the participation in convergence research may often need to be self-created through personal excitement about a specific question or collaboration, or because of potential access to research funding.

Also, disciplines can create or enforce their own culture, like many human societies, by creating their own vocabulary and vernacular for communicating their work. The vocabulary can become an intentional or unintentional barrier for members of other disciplines to integrate or collaborate.

Furthermore, disciplines have evolved their own way of collecting and sharing data. For example, Hampton et al (2013) examined how big data may impact the future of the field of ecology. Their meta-analysis indicated that, as a group, ecologists tend to design their own experiments to answer specific questions, and to a large degree do not have a culture of sharing or reusing data. Where data is shared in open databases, the vast majority was shared in genetic databases (e.g., GenBank)- genetics is a science that has been driven in many ways by a culture of data sharing.

Costs of maintaining computational infrastructure and data storage.

The amount of data is increasing at an extraordinary rate. In some fields, we now collect more data in a single year than had been collected in all of human history. This is putting tremendous pressure on the capacity of universities to manage data as well as maintaining the computational capacity to analyze to support research computation. Many universities built their research computing infrastructure with heavy support from external, often federal, grants. The availability of these funds has not kept pace with the dramatically changing needs for storage and computation. Furthermore, it has been difficult for many universities to develop a sustainable business model to support the increasing capacity needed for data storage and computation. Part of the challenge with storage is deciding what data needs to be kept. Physical libraries, at least that we know of, do not save and catalogue every document ever written, at least partly because there is no business or operational model that would allow that approach to be functional. We think that decisions will need to be made regarding benefit and cost to ensure optimal storage and use of data, and the criteria that can be applied with respect to which data should be saved.

Data science vs. statistical support.

We have both been involved in implementing initiatives to develop data science programs. A challenge that we observed is the definition of "data scientists" varies among various fields or individuals. For example, one of us was involved in facilitating the development of an institutional partnership that involved biomedical disciplines from one campus and computational disciplines from another. The biomedical researchers demonstrated great enthusiasm for the bioinformatics expertise of the computational scientists. But, when digging a little deeper, some of the biomedical scientists were excited because they viewed the strength in bioinformacticists as a way to acquire help analyzing their data - essentially looking for statistical support of their research. This was a mismatch for the data scientists who focused their research on the development of new tools and/or the fundamental science of data analysis. This created tension in the partnership. Although this occurred several years ago, the term "data scientist" still means different things to different people, and this can inhibit the formation of teams participating in convergence research. 
Correlation/predictive analytics vs cause/mechanism.

Both of us in our current roles as provost (Coleman) and vice chancellor for research (Sui) are using big data and predictive analytics to ask questions and to help drive resource allocation. These tools are powerful. But we also worry that the rapidly growing ability to correlate and tease apart how different variables are related to each other can lead to inappropriate conclusions regarding causality. It can be easy to mistake correlation for causality.

One humorous example of the potential to confuse correlation and causality is the predictive value of ice cream consumption for weight gain over an annual period. A big data analysis would show that ice cream consumption peaks during summer months in the US and declines in winter months. Alternatively, weight gain follows an almost exact opposite pattern. Weight gain in US population reaches a peak in winter months and reaches its low point in summer. Thus, it turns out that ice cream consumption is in fact a great correlative (inverse) predictor of weight gain over the course of a year - the higher the ice cream consumption the lower the weight gain. This is a wonderful conclusion for those of us who love ice cream. But, unfortunately, although ice cream consumption over the course of a year is a great inverse predictor of weight gain over a year, it has no causal relationship. Both curves are driven by seasonal temperatures and culture.

As it becomes easier and easier to construct predictive analysis relating variables, it will also become more and more likely that causation and correlation can become confused. This can easily lead to bad decisions on policy or resource allocation. Furthermore, transdisciplinary research and team science always increase the difficulty and complexity of reproducibility and replicability. Also, managing large teams in research projects entails new human dynamics, and not all teams succeed admirably. In fact, some teams end in catastrophic failures.

Some strategies we have employed.

Both of us have been involved in implementing several strategies to facilitate interdisciplinary, transdisciplinary, and convergence research in our various roles. The results of these various strategies have been either mixed or hard to decipher. None of these strategies were outright failures. In most every case, the strategies facilitated positive outcomes. The challenge is determining whether the strategy really facilitated changing the culture of research, or whether the strategies produced positive outcomes enhancing research infrastructure or supporting the most highly productive, energetic, and/or ambitious faculty. And, it is also hard to determine whether the resources allocated to these initiatives created the highest ROI with respect to increasing team-based research. We list some strategies that we were involved in implementing along with links to websites describing some of them for those interested in further information:

a. Designing research facilities to facilitate convergence research. Examples that we were involved with: Bond Life Sciences Center, University of Missouri, https:// bondlsc.missouri.edu/and Bioscience Research Collaborative, Rice University, https://brc.rice.edu. By any measure, the researchers housed in these facilities have been successful, and that many of the researchers work in collaborative teams. These state-ofthe-art of facilities provided great 
environments for productive researchers.

b. Seed grant programs to support interdisciplinary or convergence research: A Chancellor's Research and Innovation Fund, funded partially through athletics revenue, was created at the University of Arkansas, https:// chancellorsfund. uark.edu/innovation-and-collaboration. This program has seeded several collaborations in its first three years, and some seed grants have led to successful large collaborative research proposals. We have focused the resources on new collaborations. We should point out, though, the program has not yet run long enough to determine the return on its investment.

c. Creating interdisciplinary structures that don't compete with disciplinary homes. The Bond Life Science Center was designed with a specific model around faculty lines, space, distribution of funds equivalent to indirect cost recovery, and salary savings obtained through support of salary by research grants to minimize competition between departments across campus and the center. This model at least worked at the start of the center in facilitating departments across campus moving faculty into the facility. At the Desert Research Institute (www.dri.edu), five disciplinary units (Biological Sciences, Earth Science, Energy, Atmospheric Science, and Hydrological Sciences) were combined into three larger units (Earth and Ecosystem, Hydrological Sciences, and Atmospheric Science), and the administrative savings were used to create two interdisciplinary centers, selected through a faculty review process, focused on bringing teams from across disciplines in institute together to solve larger issues. The original two centers were focused on alpine watersheds and arid lands environmental restoration. These have morphed into different areas of strength and need.

d. Interdisciplinary graduate and post-graduate programs; The University of Arkansas has six interdisciplinary graduate programs that cross department and college lines (https://graduate-and-international.uark.edu/ more-information/our-staff/interdisciplinary.php) reporting to the Graduate School and International Studies that have helped to support interdisciplinary work. At Virginia Commonwealth, several interdisciplinary Ph.D. programs were created - one that has become particularly successful and distinctive is Media, Art and Text (https://matx.vcu.edu/).

We are aware that in recent years, cluster hiring has been a common practice among multiple institutions to promote interdisciplinary collaboration. Although there are positive reports on this new practice, cluster hiring has its own problems (https://www.aplu.org/ members/commissions/urban-serving-universities/student-success/cluster.html). In addition, we believe that the global movement towards an open science paradigm has and will continue to promote convergence research and interdisciplinary collaboration despite renewed emphasis on IP protection in the U.S. and some other countries. 


\section{Summary and Conclusion}

Multiple grand challenges, ranging from dealing with global climate change and addressing the widening income and health disparity, to fighting terrorism and combating misinformation and fake news, can't be resolved by any individual discipline. More than ever, we need interdisciplinary collaboration and teamwork. Following the previous three paradigms in empirical, theoretical, and computational approaches to science, the growth of big data and data science are emerging as the fourth paradigm in the form of eScience that could potentially further facilitate convergence research, which in most cases also call for a team science approach. New insight from studies of the science of team science provide further guidance related to the composition, size, and leadership of teams. Indeed, there are no better times in the history of higher education than now to conduct convergence research through big data and team science to address grand societal challenges that transcend traditional disciplinary boundaries.

However, we do want to conclude this paper with one caveat - by emphasizing the need of convergence research and interdisciplinary collaboration, we are NOT abandoning/marginalizing the traditional discipline-based research nor in- dividual-based inquiries. In fact, we need more cutting-edge discipline-based work in order to be more productive and effective in our convergence efforts (Jacobs, 2013). Likewise, by arguing for the need of a team science approach and collaboration, we do not want to marginalize individual-based endeavors. To contrary, we believe all research must necessarily be conducted individually at some point, even for projects involving large teams. So, it is not either/or; moving forward, we need both discipline-based, individual research and convergent, team-based transdisciplinary endeavors. It has been (and continues to be) through the dialectal process of convergent/divergent, disciplinary/interdisciplinary, individual/ team-based approaches that our research enterprise has been propelled to a new level for excellence to make our world a better place for all.

\section{Acknowledgement:}

The authors gratefully acknowledge the assistance John Post and Angela Bolinger provided in the preparation of this paper. DS also acknowledges the fruitful discussions he had with his former NSF colleagues on topics related to harnessing the data revolution, growing convergence research, and team science, which planted the seeds for this paper.

\section{References}

Abbott, A. 2001. Chaos of Disciplines. Chicago, IL.: University of Chicago Press.

Atkinson, J. and Crowe, M. (eds.), 2006. Interdisciplinary Research: Diverse Approaches in Science, Technology, Health and Society. West Sussex, UK.: John Wiley \& Sons.

Bainbridge, W.S. and M. C. Roco (Eds.), 2016. Handbook of Science and Technology Convergence 1st ed. Berlin, Springer.

Bergmann, M., T. Jahn, T. Knobloch, W. Krohn, C. Pohl, and E. Schramm, 2012. Methods for Transdisciplinary Research: A Primer for Practice. Chicago, IL.: University of Chicago Press.

Börner, K., Contractor, N., Falk-Krzesinski, H.J., Fiore, S.M., Hall, K.L., Keyton, J., Spring, B., Stokols, D., Trochim, W., and Uzzi, B., 2010. A Multi-Level Systems Perspective for the Science of Team Science. Science Translational Medicine 2, 49cm24. 
Disis, M., and Slattery, J., 2010. The Road We Must Take: Multidisciplinary Team Science. Science Translational Medicine 2, 22cm29.

Dobson, J.E., 2019. Critical Digital Humanities: The search for a methodology. Urbana, IL.: University of Illinois Press.

Falk-Krzesinski, H. J., Contractor, N., Fiore, S. M., Hall, K. L., Kane, C., Keyton, J., Klein, J.T., Spring, B., Stokols, D. \& Trochim, W. (in press). Mapping a Research Agenda for the Science of Team Science. Research Evaluation.

Fiore, S.M., 2008. Interdisciplinarity as teamwork-How the science of teams can inform team science. Small Group Research 39, 251-277.

Frodeman, R., J. T. Klein, and C. Mitcham (eds.), 2010. The Oxford Handbook of Interdisciplinarity. New York, N.Y.: Oxford University Press.

Gold, M.K. and L. F. Klein (eds.), 2019. Debates in the Digital Humanities. Minneapolis, MN.: Univ Of Minnesota Press.

Graff, H. J. 2015. Undisciplining Knowledge. Baltimore, MD.: Johns Hopkins University Press.

Hampton, S.E., C. A. Strasser, J. J. Tewksbury, W. K. Gram, A. E. Budden, A. L. Batcheller, C. S. Duke, and J. H. Porter, 2013. Big data and the future of ecology. Frontiers in Ecology and Environment 11(3): 156-162.

Hey, T., Tansley, S., \& Tolle, K. (Eds.), 2010. The Fourth Paradigm: Data-Intensive Scientific Discovery. Microsoft Research, available on-line at: https://www.microsoft. com/en-us/research/publication/fourth-paradigm-data-intensive-scientific-discovery.

Jacobs, J. A., 2013. In Defense of Disciplines: Interdisciplinarity and Specialization in the Research University. Chicago, IL.: University of Chicago Press.

King, G. and N. Persily, 2019. A new model for industry-academic partnerships. PS: Political Science and Politics. Publisher's version available on-line at http://j. $\underline{\mathrm{mp} / 2 \mathrm{q} 1 \mathrm{IQpH}}$ (last accessed on July 7, 2019).

Klein, J. T., 1990. Interdisciplinarity: History, Theory, and Practice. Detroit, MI.: Wayne State University Press.

Levit, A., 2018. Humanity Works: Merging technologies and people for the workforce of the future. New York, NY.: Kogan Page Inspire.

National Academies of Sciences (NAS), 2005a. Facilitating Interdisciplinary Research. Washington D.C., National Academies Press.

National Academies of Sciences (NAS), 2005b. Enhancing the Effectiveness of Team Science. Washington D.C., National Academies Press.

National Academies of Sciences (NAS), 2014. Convergence: Facilitating Transdisciplinary Integration of Life Sciences, Physical Sciences, Engineering, and Beyond. Washington D.C., National Academies Press.

National Academies of Sciences (NAS), 2018. The Integration of the Humanities and Arts with Sciences, Engineering, and Medicine in Higher Education: Branches from the Same Tree. Washington, DC: The National Academies Press.

Schwab, K., 2015. The Fourth Industrial Revolution. https://www.weforum.org/about/ the-fourth-industrial-revolution-by-klaus-schwab

Stokols, D., Hall, K.L., Taylor, B.K., and Moser, R.P., 2008. The Science of Team Science: Overview of the Field and Introduction to the Supplement. American Journal of Preventive Medicine 35, S77-S89. 
Szostak, R., 2013. The state of the field: Interdisciplinary research. Issues in Interdisciplinary Studies 31: 44-65

Watson, P., 2016. Convergence: The idea at the heart of science. New York, NY.: Simon \& Schuster.

Woelert, P. and V. Millar, 2013. The 'Paradox of Interdisciplinarity' in Australian Research Governance. Higher Education 66 (6): 755-767.

Wuchty, S., Jones, B.F., and Uzzi, B., 2007. The Increasing Dominance of Teams in Production of Knowledge. Science 316, 1036-1038. 